\title{
Impact of EDCs on cation transporter channel in the mouse placenta during pregnancy
}

\section{Myoungho Lee, Jae-Hwan Lee, Tran Dinh Nam, Jin Yong An and Eui-Bae Jeung}

Laboratory of Veterinary Biochemistry and Molecular Biology, Chungbuk National University, Cheongju, Chungbuk, 28644, Republic of Korea

\section{Introduction}

Placenta exchanges vital factors including oxygen, carbon dioxide, cation(copper, iron, calcium) and glucose that are essential to fetal growth. Copper, iron, calcium cation and glucose transfer genes are regulated by reproduction-related hormones, vitamin $D$ and human placental lactogen. These molecules are transferred by specific receptors located on cell membrane or cytoplasm in placenta. These substances disturb action of reproduction-related hormones (ex> estrogen, progesterone) by interacting with their receptors, or affecting the expression of transporting genes for cations.

\section{Methods}

To examine the effects of EDCs exposure during pregnancy, we conducted the in vivo model study using Pregnancy mouse. We used different doses of octyl-phenol (OP; $50 \mathrm{mg} / \mathrm{kg} / \mathrm{day}$ ), and bisphenol A (BPA; $50 \mathrm{mg} / \mathrm{kg} /$ day) in pregnancy mice for GD 11.5 16.5. Ethinyl estradiol (EE; $0.2 \mathrm{mg} / \mathrm{kg} / \mathrm{day})$, which activates estrogen receptors, was used as a positive control. ICI $182780(70 \mathrm{ug} / \mathrm{kg})$ were used with estrogen antagonist. The mRNA expressions levels of calcium transporting genes (PMCA1 and TRPV6), copper transporting genes (CTR1 and ATP7A), and iron transporting genes (IREG1 and HEPH) were quantified by qRT-PCR

\section{Result}

qRT-PCR result, mRNA and protein level of PMCA1 was decreased at EE, OP, BPA, BPA+ICI. Expression level of CTR1 and ATP7A was decreased at EE, EE+ICI, OP, BPA. Level of IREG1 mRNA and protein was decreased at $E E+I C I$. HEPH was decrease at $E E+\mid C I, O P+I C I$, BPA compared to the vehicle control.

\section{Conclusion}

We confirmed that EDCs have influences on metal ion channels such as calcium, copper, and iron transporters during the pregnancy. EDCs could affect cation transporting channel so that cause negative effect on fetal growth.

\section{Reference}

1. Sun Kim, Beum-Soo An, Hyun Yang, Eui-Bae Jeung. Effects of octylphenol and bisphenol A on the expression of calcium transport genes in the mouse duodenum and kidney during pregnancy. Toxicology 303, 99-106 (2013) 2. Marcel G.R. ter Veld, E. Zawadzkaa, Ivonne M.C.M. Rietjens, Albertinka J. Murk. Estrogenicity of foodassociated estrogenic compounds in the fetuses of female transgenic mice upon oral and IP maternal exposure. Reproductive Toxicology 27 (2), 133-139 (2009)
EP585: Endocrine Disruptors
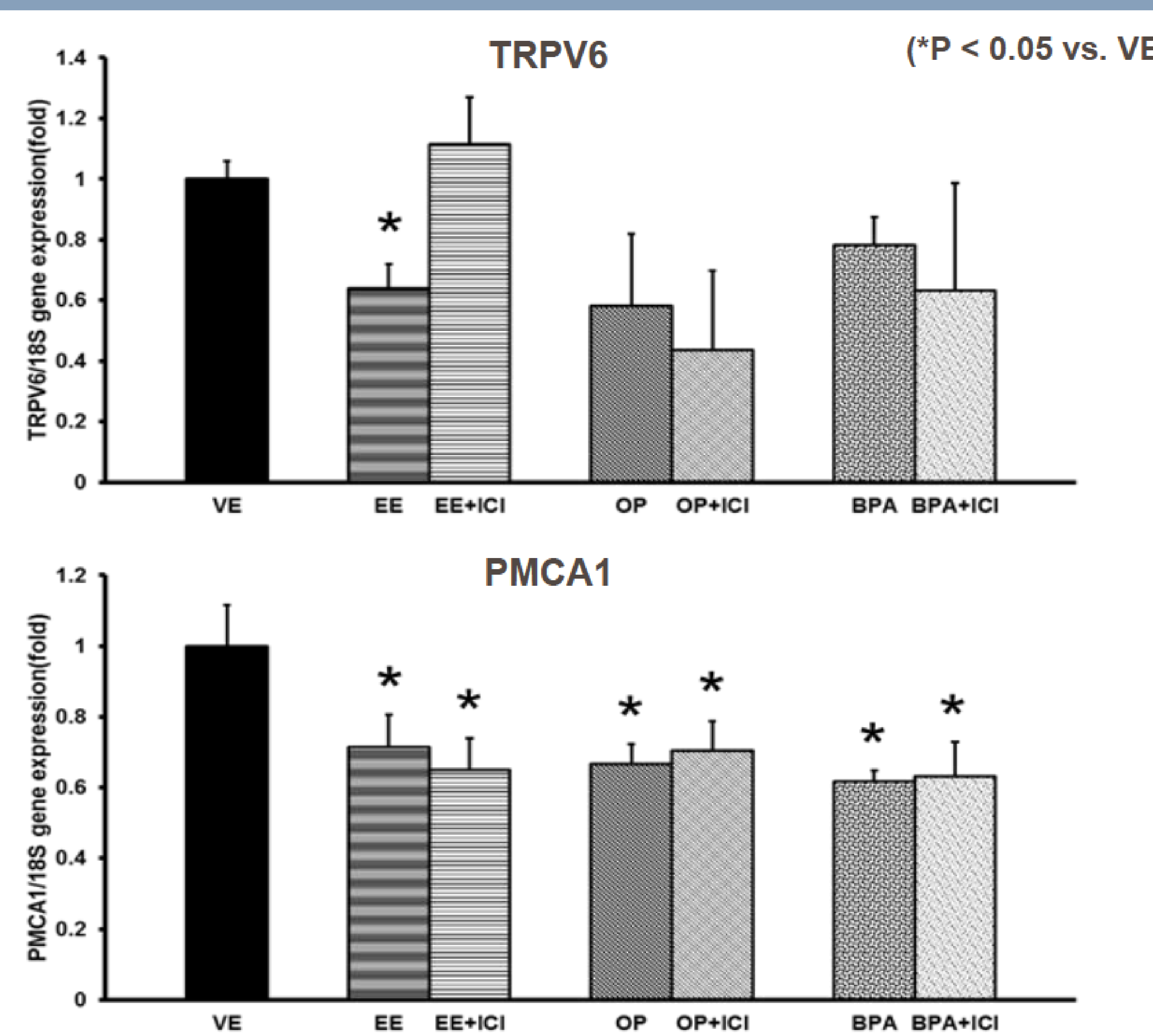

Fig 1. Calcium transporting genes expression
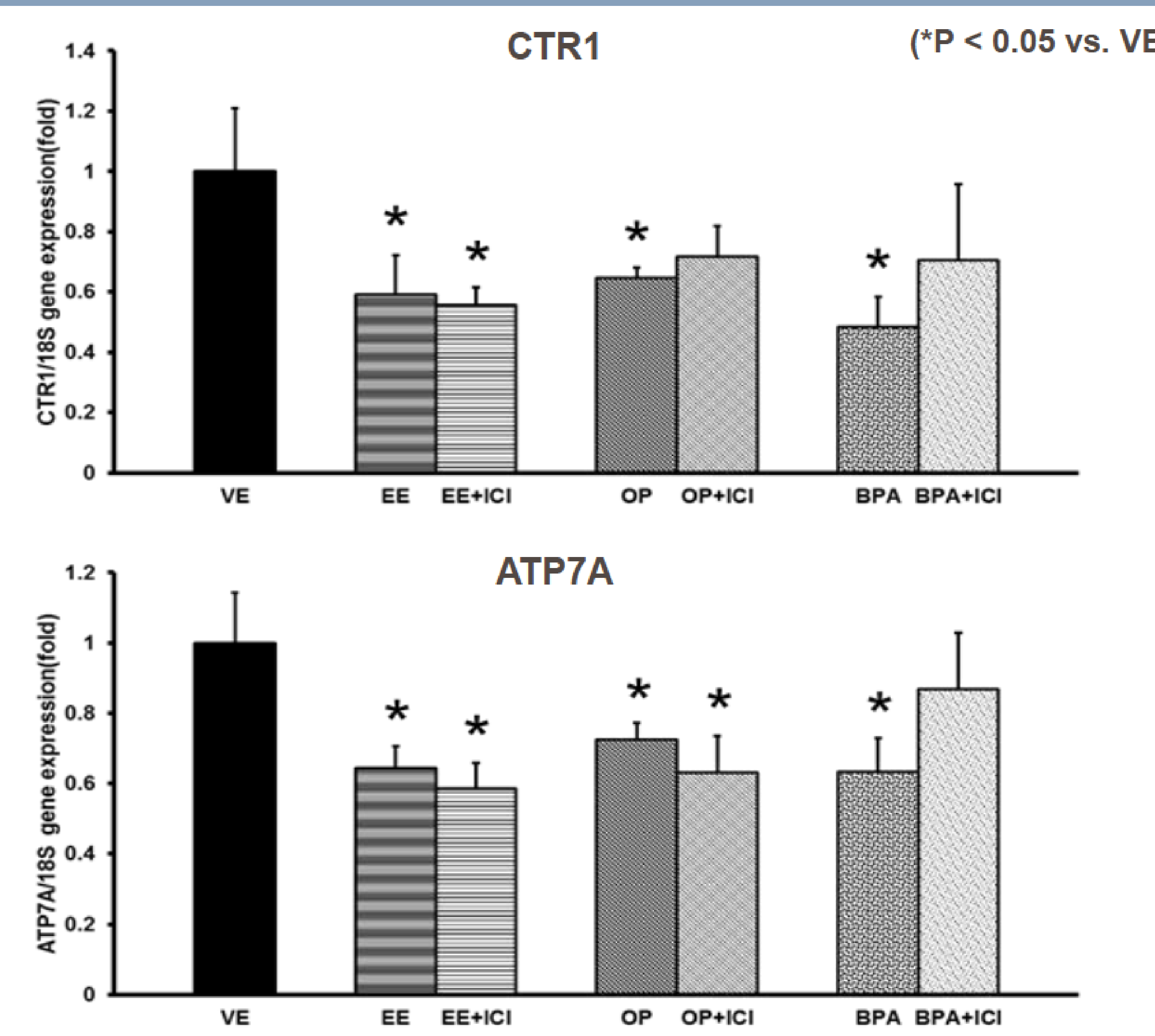

Fig 2. Copper transporting genes expression
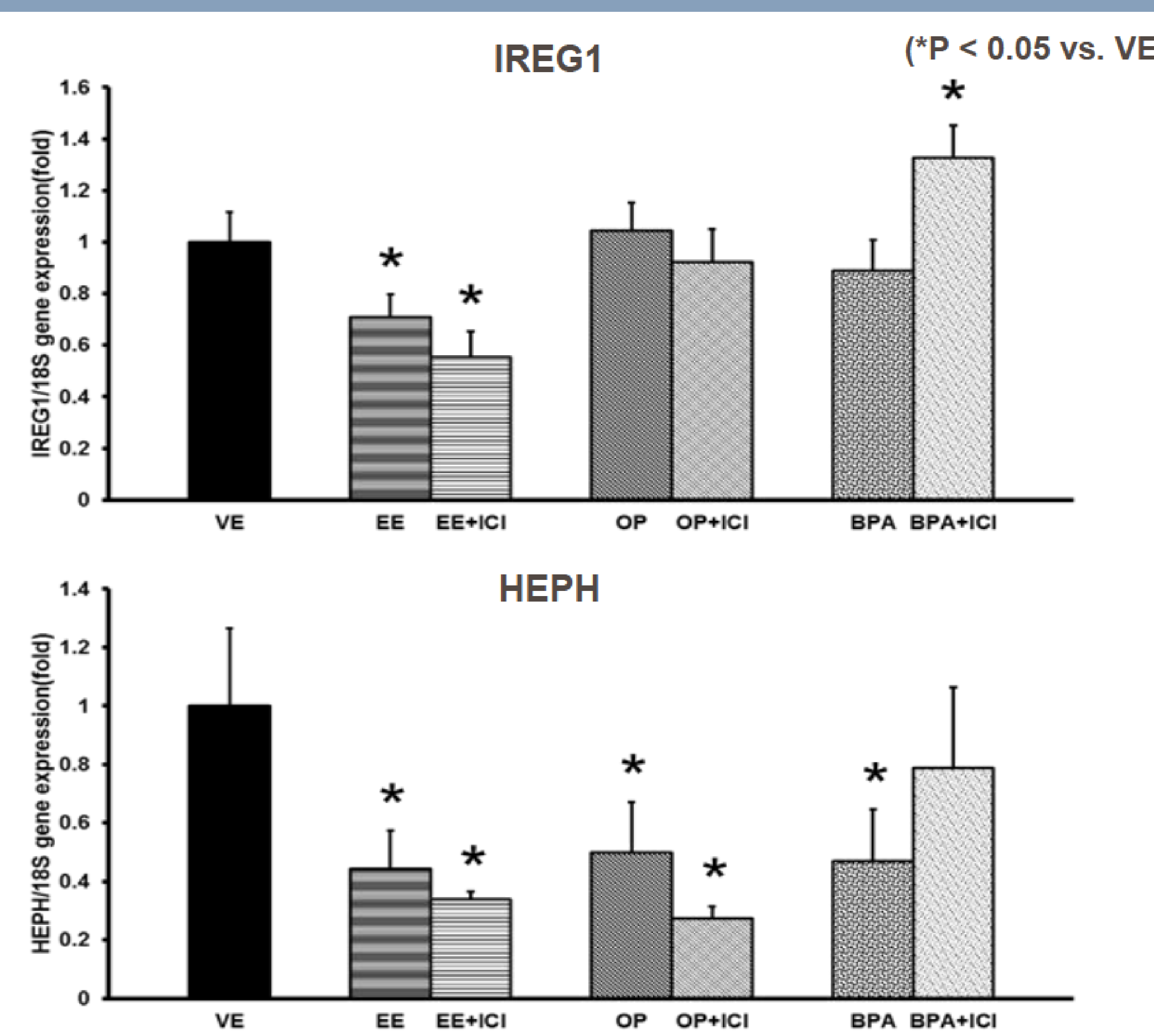

Fig 3. Iron transporting genes expression 